\title{
PERFECT POWERS IN AN ALTERNATING SUM OF CONSECUTIVE CUBES
}

\author{
Pranabesh Das, Pallab Kanti Dey, Bibekananda Maji and \\ Sudhansu SeKhar Rout \\ University of Waterloo, Canada, Indian Statistical Institute Delhi, India, \\ Indian Institute of Technology Indore, India and Institute of Mathematics \\ and Applications Bhubaneswar, India
}

\begin{abstract}
In this paper, we consider the problem about finding out perfect powers in an alternating sum of consecutive cubes. More precisely, we completely solve the Diophantine equation $(x+1)^{3}-(x+2)^{3}+\cdots-$ $(x+2 d)^{3}+(x+2 d+1)^{3}=z^{p}$, where $p$ is prime and $x, d, z$ are integers with $1 \leq d \leq 50$.
\end{abstract}

\section{INTRODUCTION}

In 1964, Leveque ([11]) proved that, if $f(x) \in \mathbb{Z}[x]$ is a polynomial of degree $k \geq 2$ with at least two simple roots, and $n \geq \max \{2,5-k\}$ is an integer, then the superelliptic equation

$$
f(x)=z^{n}
$$

has at most finitely many solutions in integers $x$ and $z$. In 1976, this result was extended by Schinzel and Tijdeman ([17]). They proved that the equation (1.1) has at most finitely many solutions in integers $x, z$ and variable $n \geq$ $\max \{2,5-k\}$ through an application of lower bounds for linear forms in logarithms.

Earlier in 1875, Lucas ([12]) considered the Diophantine equation

$$
1^{2}+2^{2}+\cdots+x^{2}=y^{2},
$$

2020 Mathematics Subject Classification. 11D61, 11D41, 11F11, 11F80.

Key words and phrases. Diophantine equation, Galois representation, Frey curve, modularity, level lowering, linear forms in logarithms. 
and claimed that $(1,1)$ and $(24,70)$ are the only solutions in positive integers $(x, y)$ to the equation (1.2). In 1918, Watson ([22]) completely solved the equation (1.2).

In 1956, Schäffer ([16]) studied the more general equation

$$
1^{k}+2^{k}+\cdots+x^{k}=y^{n} .
$$

It is easy to see that for every $k$ and $n,(x, y)=(1,1)$ is a solution of (1.3). Schäffer ([16]) proved that if $k \geq 1$ and $n \geq 2$ are fixed, then (1.3) has only finitely many solutions except the following cases

$$
(k, n) \in\{(1,2),(3,2),(3,4),(5,2)\} .
$$

In the same paper Schäffer gave a conjecture regarding the integral solutions of (1.3). He conjectured that, for $k \geq 1$ and $n \geq 2$ with $(k, n)$ not in the set (1.4), equation (1.3) has only one non-trivial solution, namely $(k, n, x, y)=(2,2,24,70)$. There are some results, at least in principle, to determine all solutions of (1.3).

Jacobson, Pintér, Walsh ([8]) confirmed the conjecture for $n=2$ and $k$ even with $k \leq 58$. Recently, Bennett, Győry, Pintér ([1]) proved completely the Schäffer conjecture for arbitrary $n$ and $k \leq 11$. As an extension of [1], Pintér ([14]) proved Schäffer conjecture for odd values of $k$ with $1 \leq k \leq 170$ and even values of $n$.

Zhang and Bai ([24]) generalized the equation (1.3) and considered the more general equation

$$
(x+1)^{k}+(x+2)^{k}+\cdots+(x+d)^{k}=y^{n},
$$

for $k \geq 2$. They completely solved the equation (1.5) for $k=2$ and $d=x$. For $k=2$, they also proved that for a prime $p \equiv \pm 5(\bmod 12)$ with $p \mid d$ and $\nu_{p}(d) \not \equiv 0(\bmod n)$, the equation (1.5) has no integer solution.

Recently, Soydan ([20]) considered the equation (1.5) for $k \geq 2$ and $d=l x$ for some integer $l \geq 2$. He proved that all solutions of the equation (1.5) in integers $x, y \geq 1$ and $n \geq 2$ satisfy $n<C$, where $C$ is an effectively computable constant depending only on $l$ and $k$. He also proved that for $k \neq 3$ all solutions of the equation (1.5) in integers $x, y, n$ with $x, y \geq 1, n \geq 2$ and $l \equiv 0(\bmod 2)$ satisfy $\max \{x, y, n\}<C_{1}$ where $C_{1}$ is an effectively computable constant depending only on $l$ and $k$.

Cassels ([5]) solved the equation (1.5) completely for $n=2, d=3$ and $k=3$. Zhang ([25]) determined the perfect powers in sum of three consecutive cubes by rewriting the equation (1.5) for $k=d=3$ as

$$
(x-1)^{3}+x^{3}+(x-1)^{3}=y^{n} .
$$

Stroeker ([21]) completely solved the equation (1.5) for $k=3, n=2$ and $2 \leq d \leq 50$ using linear forms in elliptic logarithms. Recently, Bennett, Patel and Siksek ([3]) extended the result of Stroeker for $n \geq 3$. 
Several generalizations of (1.3) have been considered by different authors. For example Dilcher ([7]) studied the equation

$$
\chi(1) 1^{k}+\chi(2) 2^{k}+\cdots+\chi(x f)(x f)^{k}=b y^{n},
$$

where $\chi$ is a primitive quadratic residue class character with conductor $f$ and $k, b \neq 0$ are fixed integers. This may be viewed as a character-twisted analogue of a classic equation of Schäffer. Recently, Bennett ([2]) completely solved the equation

$$
1^{k}-3^{k}+5^{k}-\cdots+(4 x-3)^{k}-(4 x-1)^{k}=-y^{n}
$$

for $3 \leq k \leq 6$.

In this paper we consider the following Diophantine equation

$$
(x+1)^{3}-(x+2)^{3}+\cdots+(-1)^{m-1}(x+m)^{3}=z^{p},
$$

where $m, x, z$ are integers with $m \geq 2$ and $p$ is any prime number.

We note that for a fixed ordered tuple $(m, p)$, it is easy to conclude that equation (1.9) has only finitely many solutions. Since we are dealing with an infinite collection of tuples $(m, p)$ in our case it is not obvious that there are finitely many solutions. On top of that we also find the precise solutions of equation (1.9) using a combination of both classical and modern techniques in Diophantine analysis.

Simplifying (1.9), for odd $m$ we obtain

$$
\left(x+\frac{m+1}{2}\right)\left\{\left(x+\frac{m+1}{2}\right)^{2}+3 \frac{m^{2}-1}{4}\right\}=z^{p} .
$$

Putting $m=2 d+1$ for some positive integer $d$, we have

$$
(x+d+1)\left\{(x+d+1)^{2}+3 d(d+1)\right\}=z^{p} .
$$

From the equation (1.11), we can see that $\operatorname{gcd}\left((x+d+1),(x+d+1)^{2}+3 d(d+1)\right)$ divides $3 d(d+1)$. Hence

$$
x+d+1=\alpha z_{1}{ }^{p} \quad \text { and } \quad(x+d+1)^{2}+3 d(d+1)=\beta z_{2}{ }^{p}
$$

for some integers $z_{1}, z_{2}$ and rationals $\alpha, \beta$ with $\alpha \beta=1$ and $z_{1} z_{2}=z$. The denominator and the numerator of $\alpha$ and $\beta$ are composed of prime divisors of $3 d(d+1)$. From (1.11) and (1.12), we deduce the following ternary equation

$$
\beta z_{2}^{p}-\alpha^{2} z_{1}^{2 p}=3 d(d+1) .
$$

If $\beta<0$, then from the equation (1.12), we have $z_{2}<0$. Also $\alpha<0$ as $\alpha \beta=$ 1. Hence, $\left( \pm z_{1}, z_{2}\right)$ is an integral solution of equation (1.13) corresponding to $(\alpha, \beta)$ if and only if $\left( \pm z_{1},-z_{2}\right)$ is an integral solution of equation (1.13) corresponding to $(-\alpha,-\beta)$. Therefore it is enough to solve the equation (1.13) for $\beta>0$. 
Suppose $S_{d}$ is the set of such pairs of positive rationals $(\alpha, \beta)$. We need to solve the equation (1.13) for each $(\alpha, \beta) \in S_{d}$ with $1 \leq d \leq 50$. Clearing denominators we can rewrite the equation (1.13) as

$$
r z_{2}^{p}-s z_{1}^{2 p}=t
$$

where $r, s, t$ are positive integers and $\operatorname{gcd}(r, s, t)=1$.

Now we state our main theorem as follows.

TheOREM 1.1. For $m=2 d+1$ with $1 \leq d \leq 50$, the integral solutions $(x, z, p)$ of the equation (1.9) are given in the Table 1.

REMARK 1.2. If $z=0$, then from the equation (1.11), we have $x=-(d+$ $1)$ as $(x+d+1)^{2}+3 d(d+1)>0$ for any $d$. Therefore, $(x, z, p)=(-d-1,0, p)$ are the trivial solutions of the equation (1.9) for any $d$.

REMARK 1.3. From Theorem 1.1, it is clear that for $p>7$, there is no integral solution for the equation (1.9). For $p=5,(d, x, z)=(20,-15,6)$ is the only integral solution for the equation (1.9). For $p=7,(d, x, z) \in$ $\{(4,-3,2),(15,-13,3),(27,26,6)\}$.

\section{Preliminaries}

We use well known tools such as linear forms in two logarithms, variation of Kraus criterion, modular method, local solubility, descent to prove Theorem 1.1. In this section we provide the necessary details for these methods.

Linear forms in 2 logarithms: We state a special case of the following well known result of Laurent ([10]).

Proposition 2.1 ([10, Corollary 2]). Let $\alpha_{1}$ and $\alpha_{2}$ be two positive real, multiplicatively independent algebraic numbers and $\log \alpha_{1}, \log \alpha_{2}$ be any fixed determinations of the logarithms that are real and positive. Write $D=\left[\mathbb{Q}\left(\alpha_{1}, \alpha_{2}\right): \mathbb{Q}\right]$ and

$$
b^{\prime}=\frac{b_{1}}{D \log A_{2}}+\frac{b_{2}}{D \log A_{1}}
$$

where $b_{1}, b_{2}$ are positive integers and $A_{1}, A_{2}$ are real numbers greater than one such that

$$
\log A_{i} \geq \max \left\{h\left(\alpha_{i}\right), \frac{\left|\log \alpha_{i}\right|}{D}, \frac{1}{D}\right\}, \quad i=1,2
$$

with

$$
h(\alpha)=\frac{1}{d}\left(\log |a|+\sum_{i=1}^{d} \log \max \left(1,\left|\alpha^{(i)}\right|\right)\right),
$$

where $a$ is the leading coefficient of the minimal polynomial of $\alpha$ and the $\alpha^{(i)}$ 's are the conjugates of $\alpha$ in $\mathbb{C}$. 
Let $\Lambda=b_{2} \log \alpha_{2}-b_{1} \log \alpha_{1}$. Then

$$
\log |\Lambda| \geq-25.2 D^{4}\left(\max \left\{\log b^{\prime}+0.38,10 / D, 1\right\}\right)^{2} \log A_{1} \log A_{2} .
$$

Variation of Kraus Criterion: Now we state the following variation of Kraus criterion for the non-existence of integral solutions to the equation (1.14) for given $r, s, t$ and $p$.

\begin{tabular}{||l|l||}
\hline$d$ & $(x, z, p)$ \\
\hline \hline$d$ & $(-d-1,0, p)$ \\
\hline 2 & $(0, \pm 9,2),(3, \pm 18,2),(69, \pm 612,2)$ \\
\hline 4 & $(-3,2,7),(1, \pm 24,2),(5, \pm 40,2),(235, \pm 3720,2)$ \\
\hline 5 & $(34, \pm 260,2)$ \\
\hline 6 & $(0, \pm 35,2),(11, \pm 90,2)$ \\
\hline 7 & $(-7, \pm 13,2),(160, \pm 2184)$ \\
\hline 8 & $(16, \pm 145,2)$ \\
\hline 11 & $(36, \pm 360,2)$ \\
\hline 12 & $(-9, \pm 44,2),(0, \pm 91,2),(23, \pm 252,2),(104, \pm 1287,2),(195, \pm 3016,2)$ \\
\hline 15 & $(-13,3,7)$ \\
\hline 16 & $(83, \pm 1040,2)$ \\
\hline 19 & $(-16, \pm 68,2),(-14, \pm 84,2),(34, \pm 468,2)$, \\
\hline 20 & $(170, \pm 2660,2),(265, \pm 4845,2),(5746, \pm 437844,2)$ \\
\hline 26 & $(-39,6,5),(0, \pm 189,2),(39, \pm 540,2)$ \\
\hline 27 & $(-10, \pm 216,2),(-46,-36,3),(-34,-24,3),(-22,24,3),(-10,36,3)$ \\
\hline 28 & $(13, \pm 420,2),(29, \pm 580,2)$ \\
\hline 29 & $(-24, \pm 126,2),(405, \pm 9135,2)$ \\
\hline 30 & $(-21, \pm 170,2),(0, \pm 341,2),(59, \pm 990,2)$, \\
& $(248, \pm 4743,2),(1179, \pm 42130,2),(5208, \pm 379223,2)$ \\
\hline 32 & $(-24, \pm 171,2),(319, \pm 6688,2)$ \\
\hline 34 & $(16, \pm 561,2),(35, \pm 770,2),(14245, \pm 1706460,2)$ \\
\hline 36 & $(-91,-72,3),(-39,-20,3),(-35,20,3),(17,72,3)$ \\
\hline 38 & $(2811, \pm 152190,2)$ \\
\hline 39 & $(-31, \pm 207,2),(81, \pm 1529,2),(480, \pm 11960,2)$ \\
\hline 42 & $(-124,-99,3),(0, \pm 559,2),(83, \pm 1638,2),(38,99,3)$ \\
\hline 45 & $(8, \pm 702,2),(69, \pm 1495,2),(440, \pm 10854,2)$ \\
\hline 47 & $(-36, \pm 288,2),(516, \pm 13536,2)$ \\
\hline 49 & $(230, \pm 4900,2),(-95,-75,3),(-5,75,3)$ \\
\hline & \\
\hline &
\end{tabular}

TABLE 1. The integral solutions to equation (1.9) for $m=$ $2 d+1$ with $1 \leq d \leq 50$ and $p$ is prime. 
LEMma 2.2 ([3, Lemma 6.1]). Consider the equation (1.14) for $p \geq 3$. Also let $q=2 k p+1$ be a prime that does not divide $r$. Define

$$
\mu(p, q)=\left\{\eta^{2 p}: \eta \in \mathbb{F}_{q}\right\}=\{0\} \cup\left\{\zeta \in \mathbb{F}_{q}^{*}: \zeta^{k}=1\right\}
$$

and

$$
B(p, q)=\left\{\zeta \in \mu(p, q):((s \zeta+t) / r)^{2 k} \in\{0,1\}\right\} .
$$

If $B(p, q)=\emptyset$, then the equation (1.14) does not have any integral solution.

Modular method: Before going to our problem we would like to give a brief description about modular method. Let $E$ be an elliptic curve over $\mathbb{Q}$ of conductor $N$ and $\# E\left(\mathbb{F}_{q}\right)$ be the number of points on $E$ over the finite field $\mathbb{F}_{q}$ for a good prime $q$. Let $a_{q}(E)=q+1-\# E\left(\mathbb{F}_{q}\right)$. By a newform $f$ of level $N$, we mean a normalized cusp form of weight 2 for the congruence subgroup $\Gamma_{0}(N)$. Write $f=q+\sum_{i \geq 2} c_{i} q^{i}$ and $K:=\mathbb{Q}\left(c_{1}, c_{2}, \cdots\right)$ is the totally real number field generated by the Fourier coefficients of $f$.

We say that the curve $E$ arises modulo $p$ from the newform $f$ (and write $E \sim_{p} f$ ) if there is a prime ideal $\mathfrak{p}$ of $K$ above $p$ such that for all but finitely many primes $q$, we have $a_{q}(E) \equiv c_{q}(\bmod \mathfrak{p})$. If $f$ is a rational newform, then $f$ corresponds to some elliptic curve $F$ (say). If $E$ arises modulo $p$ from $f$, then also we say that $E$ arises modulo $p$ from $F$. In this regard we have the following result.

Proposition 2.3 ([6, Prop 15.2.2]). Let $E$ and $F$ be elliptic curves over $\mathbb{Q}$ with conductors $N$ and $N^{\prime}$ respectively. Suppose that $E$ arises modulo $p$ from $F$. For all primes $q$

1. if $q \nmid N N^{\prime}$, then $a_{q}(E) \equiv a_{q}(F)(\bmod p)$ and

2. if $q \nmid N^{\prime}$ and $q \| N$, then $q+1 \equiv \pm a_{q}(F)(\bmod p)$.

The following result provides a bound for the exponent $p$.

Proposition 2.4 ([6, Prop 15.4.1]). Let $E / \mathbb{Q}$ be an elliptic curve of conductor $N$ with $h \mid \# E(\mathbb{Q})_{\text {tors }}$ for some integer $h$. Suppose $f$ is a newform of level $N^{\prime}$ and $q$ be a prime with $q \nmid N^{\prime}, q^{2} \nmid N$. Also let

$$
T_{q}=\{a \in \mathbb{Z}:-2 \sqrt{q} \leq a \leq 2 \sqrt{q}, a \equiv q+1(\bmod h)\} .
$$

Let $c_{q}$ be the $q$-th coefficient of $f$ and define

$$
B_{q}^{\prime}(f):=\operatorname{Norm}_{K / \mathbb{Q}}\left((q+1)^{2}-c_{q}^{2}\right) \prod_{a \in T_{q}} \operatorname{Norm}_{K / \mathbb{Q}}\left(a-c_{q}\right)
$$

and

$$
B_{q}(f)= \begin{cases}q \cdot B_{q}^{\prime}(f) & \text { if } f \text { is irrational, } \\ B_{q}^{\prime}(f) & \text { if } f \text { is rational. }\end{cases}
$$

If $E \sim_{p} f$, then $p \mid B_{q}(f)$. 
Descent: Following well known method is very useful to eliminate possible integral solution for a Diophantine equation of certain type.

Consider the equation in integers $R, X, S, Y, T$,

$$
R Y^{p}-S X^{2 p}=T
$$

with $R, S, T$ pairwise coprime integers.

For a prime $q$, we define

$$
S^{\prime}:=\prod_{\operatorname{ord}_{q}(S) \text { is odd }} q .
$$

Then $S S^{\prime}=v^{2}$ for some integer $v$. Take $R S^{\prime}=u$ and $T S^{\prime}=m n^{2}$ for some integers $u, m$ and $n$ with $m$ squarefree. Substituting these values in the equation (2.3), we have

$$
\left(v X^{p}+n \sqrt{-m}\right)\left(v X^{p}-n \sqrt{-m}\right)=u Y^{p} .
$$

Let $K=\mathbb{Q}(\sqrt{-m})$ and $\mathcal{O}$ be its ring of integers. Let $P$ be the set of prime ideals of $\mathcal{O}$ which divide $u$ and $2 n \sqrt{-m}$. The $p$-Selmer group is given by

$$
K(P, p)=\left\{\epsilon \in K^{*} / K^{* p}: \operatorname{ord}_{\mathcal{P}}(\epsilon) \equiv 0(\bmod p) \text { for } \mathcal{P} \notin P\right\}
$$

and this is a $\mathbb{F}_{p}$-vector space of finite dimension. Let

$$
\Theta=\left\{\epsilon \in K(P, p): \operatorname{Norm}(\epsilon) / u \in \mathbb{Q}^{* p}\right\} .
$$

Now it is easy to see that

$$
v X^{p}+n \sqrt{-m}=\epsilon Z^{p},
$$

where $\epsilon \in \Theta$ and $Z \in K^{*}$.

Lemma 2.5 ([3, Lemma 9.1]). Let $\mathfrak{q}$ be a prime ideal of $K$. Suppose one of the following holds:

1. $\operatorname{ord}_{\mathfrak{q}}(v), \operatorname{ord}_{\mathfrak{q}}(n \sqrt{-m}), \operatorname{ord}_{\mathfrak{q}}(\epsilon)$ are pairwise distinct modulo $p$;

2. $\operatorname{ord}_{\mathfrak{q}}(2 v), \operatorname{ord}_{\mathfrak{q}}(\epsilon), \operatorname{ord}_{\mathfrak{q}}(\bar{\epsilon})$ are pairwise distinct modulo $p$;

3. $\operatorname{ord}_{\mathfrak{q}}(2 n \sqrt{-m}), \operatorname{ord}_{\mathfrak{q}}(\epsilon), \operatorname{ord}_{\mathfrak{q}}(\bar{\epsilon})$ are pairwise distinct modulo $p$. Then there is no $X \in \mathbb{Z}$ and $Z \in K$ satisfying the equation (2.4).

Lemma 2.6 ([3, Lemma 9.2]). Let $q=2 k p+1$ be a prime. Suppose $q \mathcal{O}=\mathfrak{q}_{1} \mathfrak{q}_{2}$ where $\mathfrak{q}_{1}, \mathfrak{q}_{2}$ are distinct prime ideals in $\mathcal{O}$, such that $\operatorname{ord}_{\mathfrak{q}_{j}}(\epsilon)=0$ for $j=1,2$. Let

$$
\chi(p, q)=\left\{\eta^{p}: \eta \in \mathbb{F}_{q}\right\} .
$$

Let

$C(p, q)=\left\{\zeta \in \chi(p, q):((v \zeta+n \sqrt{-m}) / \epsilon)^{2 k} \equiv 0\right.$ or $1\left(\bmod \mathfrak{q}_{j}\right)$ for $\left.j=1,2\right\}$.

Suppose $C(p, q)=\emptyset$. Then there is no $X \in \mathbb{Z}$ and $Z \in K$ satisfying the equation (2.4). 
Lemma 2.7 ([3, Lemma 9.3]). Suppose

1. $\operatorname{ord}_{\mathfrak{q}}(n \sqrt{-m})<p$ for all prime ideals $\mathfrak{q}$ of $\mathcal{O}$;

2. the polynomial $U^{p}+(\rho-U)^{p}-2$ has no roots in $\mathcal{O}$ for $\rho=1,-1,-2$;

3. the only root of the polynomial $U^{p}+(2-U)^{p}-2$ in $\mathcal{O}$ is $U=1$.

Then, for $\epsilon=n \sqrt{-m}$, the only solution to equation (2.4) with $X \in \mathbb{Z}$ and $Z \in K$ is $X=0$ and $Z=1$.

\section{Proof of Theorem 1.1 For $p \geq 5$}

At first, we use lower bounds for linear forms in two logarithms to bound the exponent $p$ appearing in (1.13).

Lemma 3.1. Let $p>19$. Consider

$$
\alpha_{1}=\beta / \alpha^{2} \quad \text { and } \quad \alpha_{2}=z_{1}^{2} / z_{2}(\neq 1)
$$

with $\left|z_{1}\right| \geq 2$ and $z_{2} \geq 2$.

Then $\alpha_{1}$ and $\alpha_{2}$ are positive and multiplicatively independent. Moreover, if we write

$$
\Lambda=\log \alpha_{1}-p \log \alpha_{2}
$$

then

$$
0<\Lambda<\frac{3 d(d+1)}{\alpha^{2} z_{1}^{2 p}}
$$

Proof. One can see that $\alpha_{1}$ and $\alpha_{2}$ are positive as $\beta>0$ and $z_{2}>0$. From the equations (1.13),(3.1) and (3.2), we have

$$
e^{\Lambda}-1=\frac{\beta z_{2}^{p}}{\alpha^{2} z_{1}^{2 p}}-1=\frac{3 d(d+1)}{\alpha^{2} z_{1}^{2 p}}>0 .
$$

Therefore $0<\Lambda<\frac{3 d(d+1)}{\alpha^{2} z_{1}^{2 p}}$ since $e^{x}-1>x$ for any positive real number $x$.

Now we want to prove that $\alpha_{1}$ and $\alpha_{2}$ are multiplicatively independent. On contrary, let us suppose that $\alpha_{1}$ and $\alpha_{2}$ are not multiplicatively independent i.e., there exist coprime positive integers $a$ and $b$ such that $\alpha_{1}^{a}=\alpha_{2}^{b}$. Clearly $\alpha_{1} \neq 1$. Then $a \operatorname{ord}_{l}\left(\alpha_{1}\right)=b \operatorname{ord}_{l}\left(\alpha_{2}\right)$ for all prime $l$. Hence $b \mid \operatorname{ord}_{l}\left(\alpha_{1}\right)$.

Let $g=\operatorname{gcd}\left\{\operatorname{ord}_{l}\left(\alpha_{1}\right): l\right.$ is prime $\}$. From (3.2), we have

$$
\Lambda=\log \alpha_{1}\left(1-p \frac{\log \alpha_{2}}{\log \alpha_{1}}\right)=\left|\log \alpha_{1}\right|\left|1-p \frac{a}{b}\right| .
$$

Hence from (3.3) and (3.4), we have

$$
0<\frac{1}{g} \leq\left|1-p \frac{a}{b}\right|<\frac{3 d(d+1)}{\left|\log \alpha_{1}\right| \alpha^{2} z_{1}^{2 p}}
$$

as $b \mid g$. 
Since $\left|z_{1}\right| \geq 2$, from the equation (3.5), it follows that

$$
4^{p} \leq z_{1}^{2 p}<\frac{3 d(d+1) g}{\left|\log \alpha_{1}\right| \alpha^{2}} .
$$

Therefore,

$$
p \leq \log \left(\frac{3 d(d+1) g}{\left|\log \alpha_{1}\right| \alpha^{2}}\right) / \log 4 .
$$

We wrote a Magma script ([4]) to compute this bound on $p$ for $1 \leq d \leq$ 50. The maximum possible value for the right-hand side of (3.6) is 18.11 corresponding to $d=48$ and $(\alpha, \beta)=(1 / 7056,7056)$, which is not possible as $p>19$. This completes the proof of lemma.

Lemma 3.2. Let $p>1000$. Consider

$$
\alpha_{1}=\beta / \alpha^{2} \quad \text { and } \quad \alpha_{2}=z_{1}^{2} / z_{2}(\neq 1)
$$

with $\left|z_{1}\right| \geq 2$ and $z_{2} \geq 2$. Then we have

$$
\frac{\log z_{2}}{\log z_{1}^{2}} \leq 1.01 \text {. }
$$

Proof. From the equations (3.1), (3.2) and (3.3), we have

$$
\log \alpha_{1}-p\left(\log z_{1}^{2}-\log z_{2}\right)<\frac{3 d(d+1)}{\alpha^{2} 4^{p}} .
$$

Hence

$$
\begin{aligned}
\frac{\log z_{2}}{\log z_{1}^{2}} & \leq 1+\frac{1}{p \log z_{1}^{2}}\left(\frac{3 d(d+1)}{\alpha^{2} 4^{p}}+\left|\log \alpha_{1}\right|\right) \\
& \leq 1+\frac{1}{1000 \log 4}\left(\frac{3 d(d+1)}{\alpha^{2} 4^{1000}}+\left|\log \alpha_{1}\right|\right)
\end{aligned}
$$

where $p>1000$ and $z_{2} \geq 2$. We write a Magma script ([4]) to find the maximum possible value of the right-hand side which is 1.01 , corresponding to $d=50$ and $(\alpha, \beta)=(7650,1 / 7650)$. This completes the proof.

Now we are ready to apply Proposition 2.1 to find an upper bound for $p$.

LEMMA 3.3. Let $\left(z_{1}, z_{2}\right)$ be an integral solution of the equation (1.13) with $\left|z_{1}\right|, z_{2} \geq 2$ and $z_{1}^{2} \neq z_{2}$, where $1 \leq d \leq 50$ and $(\alpha, \beta) \in S_{d}$. Then we have $p<4 \times 10^{4}$.

Proof. Let $A_{1}=\max \left\{H\left(\alpha_{1}\right), e\right\}$, where $H(a / b)=\max \{|a|,|b|\}$ for $\alpha_{1}=$ $\frac{a}{b}$. Let $A_{2}=\max \left\{z_{1}^{2}, z_{2}\right\}$. From Lemma 3.1, it is clear that the hypothesis of Proposition 2.1 is satisfied for our choices of $\alpha_{1}, \alpha_{2}, A_{1}, A_{2}$ with $D=1$. Let

$$
b^{\prime}=\frac{1}{\log A_{2}}+\frac{p}{\log A_{1}} \text {. }
$$


For $p>1000$, we have $b^{\prime}>\frac{1000}{\log A_{1}}$. For $1 \leq d \leq 50$ and $(\alpha, \beta) \in S_{d}$, the lower bound for $1000 / \log A_{1}$ is 37.27 corresponding to $d=50$ and $(\alpha, \beta)=$ (7650,1/7650). Now applying Proposition 2.1, we have

$$
\log |\Lambda| \geq-25.2\left(\max \left\{\log b^{\prime}+0.38,10,1\right\}\right)^{2} \log A_{1} \log A_{2} .
$$

Further, this gives

$$
\begin{aligned}
-\log \Lambda & \leq 25.2 \log A_{1} \log A_{2}\left(\log b^{\prime}\right)^{2} \\
& \leq 25.2 \log A_{1} \log A_{2} \log ^{2}\left(\frac{p}{\log A_{1}}+\frac{1}{\log 4}\right) .
\end{aligned}
$$

From equation (3.3), we conclude

$$
p<\frac{1}{\log z_{1}^{2}}\left\{\log \left(\frac{3 d(d+1)}{\alpha^{2}}\right)+25.2 \log A_{1} \log A_{2} \log ^{2}\left(\frac{p}{\log A_{1}}+\frac{1}{\log 4}\right)\right\} .
$$

As $\left|z_{1}\right| \geq 2$, from Lemma 3.2 we have

$$
p<\frac{1}{\log 4}\left\{\log \left(\frac{3 d(d+1)}{\alpha^{2}}\right)+26 \log A_{1} \log ^{2}\left(\frac{p}{\log A_{1}}+\frac{1}{\log 4}\right)\right\} .
$$

We wrote a Magma script ([4]) to obtain $p<4 \times 10^{4}$. This completes the proof of the lemma.

Let $z_{1}$ and $z_{2}$ be integral solutions of (1.13). Then by Lemmas 3.1, 3.2 and 3.3 , we found

$$
p<4 \times 10^{4}, \text { for }\left|z_{1}\right| \geq 2 \text { and } z_{2} \geq 2 \text { with } z_{1}^{2} \neq z_{2} .
$$

When $z_{1}^{2}=z_{2}$, we determine all the possible solutions for $1 \leq d \leq 50$ and these solutions $\left(z_{1}, z_{2}\right)$ are not satisfying the equation (1.13). Similarly, if $z_{1} \in\{-1,0,1\}$ or $z_{2}=1$, we determine all the possible solutions for $1 \leq d \leq 50$ and we observe that $(20,-15,6,5),(27,26,6,7)$ are the only integral solutions for $(d, x, z, p)$ satisfying the equation (1.11). Hence we conclude that the equation (1.11) has no integral solution for $p>4 \times 10^{4}$.

For $1 \leq d \leq 50,(\alpha, \beta) \in S_{d}$ and $5 \leq p \leq 4 \times 10^{4}$, we wrote a Magma script ([4]) with $k \leq 765$, that searches for a prime $q$ satisfying $q=2 k p+1 \nmid r$ such that $B(p, q)=\emptyset$.

We note that if there exist such a prime $q$ with $B(p, q)=\emptyset$, then by Lemma 2.2 the equation (1.13) has no solution for exponent $p$. This criterion fails when $\beta=3 d(d+1)$ (equivalently $r=t$ ) for which we have the trivial solution $\left(z_{1}, z_{2}\right)=(0,1)$. In addition, for $\beta \neq 3 d(d+1)$ (equivalently $r \neq t$ ) we found 1716 quintuples $(d, p, r, s, t)$ which fail to satisfy this criterion.

Now, to complete the proof of Theorem 1.1 for $p \geq 5$, we are remaining with the following cases.

1. $r=t$ and $p<4 \times 10^{4}$; 
2. $r \neq t$ and $p<4 \times 10^{4}$ consisting 1716 quintuples $(d, p, r, s, t)$.

To solve the equation (1.14) for $r=t$ and $p<4 \times 10^{4}$ we want to apply modular method. Here we use the recipes of Kraus ([9]) due to Wiles ([23]), Ribet ([15]) and Mazur ([13]).

In the case $r=t$, the equation (1.13) has a solution $\left(z_{1}, z_{2}\right)=(0,1)$. In fact, we want to show that $\left(z_{1}, z_{2}\right)=(0,1)$ is the only solution.

Since $r=t$, we have $\alpha=1 / 3 d(d+1)$ and thus the equation (1.13) will reduce to

$$
z_{2}^{p}-\frac{1}{(3 d(d+1))^{3}} z_{1}^{2 p}=1 .
$$

Let $R=\operatorname{Rad}(3 d(d+1))$. Since $z_{1}$ and $z_{2}$ are integers, we have $R \mid z_{1}$. Hence $z_{1}=R z_{3}$ for some integer $z_{3}$. Then from the equation (3.12), we have

$$
z_{2}^{p}-\frac{R^{2 p}}{(3 d(d+1))^{3}} z_{3}^{2 p}=1 .
$$

Take $T=\frac{R^{2 p}}{(3 d(d+1))^{3}}$ then the above equation becomes

$$
z_{2}^{p}-T z_{3}^{2 p}=1
$$

It is easy to see that $\operatorname{Rad}(T)=R$. Further we assume that

$$
2 p>3 \cdot \operatorname{ord}_{q}(3 d(d+1))
$$

for all odd primes $q$. We want to show that $z_{1}=0$ for the equation (3.12). On contrary, let us assume that $z_{1} \neq 0$, which implies $z_{3} \neq 0$. Also $z_{2} \neq 0$. The equation (3.13) can be written in the following form

$$
A x^{p}+B y^{p}+C z^{p}=0,
$$

where $A=-1, B=-T, C=1, x=1, y=z_{3}^{2}, z=z_{2}$ and also

$$
A x^{p} \equiv-1(\bmod 4), B y^{p} \equiv 0(\bmod 2) \text {. }
$$

Now we associate a solution $\left(z_{2}, z_{3}\right)$ to the Frey Curve

$$
E: \quad Y^{2}=X(X+1)\left(X-T z_{3}^{2 p}\right) .
$$

The Weierstrass model given in (3.15) is smooth as $z_{2} z_{3} \neq 0$. Let $E \sim_{p} f$, where $f$ is a weight 2 newform of level $N_{p}$ with $N_{p}$ is defined as follows:

$$
N_{p}= \begin{cases}R & \text { if } \operatorname{ord}_{2}(T)=0 \text { or } \geq 5 \\ \frac{R}{2} & \text { if } \operatorname{ord}_{2}(d(d+1))=2 \text { and } p=5 \\ R & \text { if } \operatorname{ord}_{2}(d(d+1))=3, p=5 \text { and } z_{3} \text { even } \\ R & \text { if } \operatorname{ord}_{2}(d(d+1))=4, p=7 \text { and } z_{3} \text { even } \\ 2^{2} R & \text { if } \operatorname{ord}_{2}(d(d+1))=4, p=7 \text { and } z_{3} \text { odd } \\ 2^{4} R & \text { if } \operatorname{ord}_{2}(d(d+1))=3, p=5 \text { and } z_{3} \text { odd }\end{cases}
$$


Suppose $f$ is rational and hence we get an elliptic curve $F$ of conductor $N_{p}$. Now we choose a prime $q=2 k p+1$ such that $q \nmid N_{p}$ and $E$ has multiplicative reduction at $q$. Then by Proposition $2.3, q+1 \equiv \pm a_{q}(F)(\bmod p)$ and this implies $4 \equiv\left(a_{q}(F)\right)^{2}(\bmod p)$ as $q \equiv 1(\bmod p)$.

Suppose that $f$ is irrational. Since $c_{q} \notin \mathbb{Q}$ for infinitely many coefficients of $f$, we have $B_{q}(f) \neq 0$ for infinitely many primes $q$. Then Proposition 2.4 allows us to obtain a bound for $p$. In fact, this bound is very small. Here we improve this bound by choosing a set of primes $\mathcal{P}=\left\{q_{1}, \ldots, q_{n}\right\}$ such that $q_{i} \nmid N_{p}$ for all $i$ and $B_{\mathcal{P}}(f)=\operatorname{gcd}\left(B_{q}(f): q \in \mathcal{P}\right)$. Thus, if $E \sim_{p} f$ then $p \mid B_{\mathcal{P}}(f)$.

From the above observations, the following lemma is very helpful to eliminate newforms of level $N_{p}$.

LEMMA 3.4. Let $1 \leq d \leq 50$. Also let $p \geq 5$ be a prime which satisfies the inequality (3.14) for all primes $q$. Let $N_{p}$ be given in (3.16). Suppose for each irrational newform $f$ of weight 2 and level $N_{p}$ there is a set of primes $\mathcal{P}$ not dividing $N_{p}$ such that $p \nmid B_{\mathcal{P}}(f)$. Suppose for every elliptic curve $F$ of conductor $N_{p}$ there is a prime $q=2 k p+1, q \nmid N_{p}$, such that

1. $B(p, q)=\{\overline{0}\}$, where $B(p, q)$ is in statement of Lemma 2.2;

2. $p \nmid\left(a_{q}(F)^{2}-4\right)$.

Then the equation (1.11) has only one solution with

$$
(\alpha, \beta)=\left(\frac{1}{3 d(d+1)}, 3 d(d+1)\right)
$$

satisfying $x=-(d+1)$.

Proof. If $z_{1}=0$, then we see that $x=-(d+1)$. Let us assume that, $z_{1} \neq 0$. We know that, there is a newform $f$ of level $N_{p}$ such that $E \sim_{p} f$, where $E$ is the Frey-Hellegouarch curve. If $f$ is irrational, then $p \mid B_{\mathcal{P}}(f)$, which is a contradiction to our hypothesis. Hence $f$ is rational and so $E \sim{ }_{p} F$, where $F$ is an elliptic curve of conductor $N$.

From equation (1.14), we see that $z_{2}{ }^{p}=\frac{s z_{1}{ }^{2 p}+t}{r}$. Hence

$$
\left(\frac{s z_{1}^{2 p}+t}{r}\right)^{2 k} \equiv z_{2}^{2 k p} \equiv z_{2}^{q-1} \quad(\bmod q) \text {. }
$$

Since $z_{2}{ }^{q-1} \equiv 0$ or $1(\bmod q)$, by the definition of $B(p, q)$ we have $\overline{z_{1}} \in$ $B(p, q)$. Thus, by condition $(1), \overline{z_{1}}=\overline{0}$. Hence we see that, $q \mid z_{1}$. Since $z_{1}=R z_{3}$, we have $q \mid z_{3}$. Thus, it follows that $E$ has multiplicative reduction at $q$. Hence $q+1 \equiv \pm a_{q}(F)(\bmod p)$. Since $q \equiv 1(\bmod p)$, we observe that $\left(a_{q}(F)\right)^{2} \equiv 4(\bmod p)$, which is a contradiction by condition $(2)$.

Now we wrote a Magma script ([4]) for each $1 \leq d \leq 50$ which computes the newforms of weight 2 and level $N_{p}$. Here we assume that $\mathcal{P}$ is the set of primes $<100$ which do not divide $N_{p}$. Then for each irrational newform we compute $B_{\mathcal{P}}(f)$. 
For every prime $5 \leq p<4 \times 10^{4}$ that does not divide $B_{\mathcal{P}}(f)$, satisfies the inequality (3.14) and for every isogeny class of elliptic curves $F$ of conductor $N_{p}$, we search for the primes $q=2 k p+1, q \nmid N_{p}$ with $k \leq 765$ such that conditions (1) and (2) of Lemma 3.4 hold.

If we find such a prime then the equation (1.11) has no solution with $r=t$. The criterion holds for all values of $p$ except for few small values of $p$. When $N_{p}=R$, there are 55 cases where either $p$ does not satisfy the inequality (3.14), or it divides $B_{\mathcal{P}}(f)$ for some irrational newform $f$, or $q$ does not satisfy conditions (1) and (2) of Lemma 3.4.

For other special cases of $N_{p}$ we are remaining with 3 equations, which do not satisfy the above conditions. The largest value of $p$ among the 58 quintuples is $p=19$ with

$$
d=37, \alpha=1 / 4218, \beta=4218, r=t=75044648232, s=1 .
$$

Now we have total $1716+55+3=1774$ remaining equations, which can not be eliminated by Lemma (2.2) and modular approach. These equations are of the form (1.14) with $r, s$ and $t$ positive integers and $\operatorname{gcd}(r, s, t)=1$. There is a possibility that $r, s$ and $t$ may not be pairwise coprime. We apply the procedure mentioned in [3, section 9.1] which is nothing but a repetitive way of clearing out the common factor to get an equation of the form

$$
R Y^{p}-S X^{2 p}=T
$$

where $R, S, T$ are pairwise coprime and $X, Y$ are divisors of $z_{1}, z_{2}$ respectively.

If there exist a solution for the equation(3.17), then $-S T$ is a square modulo $q$ for any odd prime $q \nmid R$. Also we check for local solubility at the primes dividing $R, S, T$, and the primes $q \leq 19$. Applying these above tests, we are remaining with 175 equations after elimination. For these remaining equations we apply descent.

By applying Lemmas 2.5 and 2.6 to the remaining equations, which were left after local solubility, we eliminate $\epsilon \in \Theta$. But we know that if $r=t$ then the equation (1.14) has a solution, i.e., $\left(z_{1}, z_{2}\right)=(0,1)$. For $r=t$, the reduction process leads to $R=T=1$. Thus the solution $\left(z_{1}, z_{2}\right)=(0,1)$ in (1.14) corresponds to $(X, Y)=(0,1)$ in (3.17). Also

$$
n \sqrt{-m}\left(K^{*}\right)^{p} \in \Theta .
$$

Hence using Lemmas 2.5 and 2.6, we eliminate all $\epsilon$ except the case $\epsilon=n \sqrt{-m}$ as the equation $(2.4)$ has a solution $(X, Z)=(0,1)$.

For the case $\epsilon=n \sqrt{-m}$, the equation (3.17) has only one solution $(X, Y)=(0,1)$ by Lemma 2.7. If $X=0$ then $z_{1}=0$ and hence, $x=-(d+1)$. If Lemmas 2.5, 2.6 and 2.7 allow us to conclude $X=0$, then we can eliminate $(r, s, t)$ as we can consider $x \neq-(d+1)$. We write a Magma script ([4]) for above procedure and we eliminate 164 equations. Now we have to solve only 11 remaining equations by Thue approach. By writing $V=Y^{2}$ in (3.17), we 
obtain the Thue equation

$$
R Y^{p}-S V^{p}=T \text {. }
$$

Using Thue equation solver in Magma ([4]), we solve the remaining equations. Finally we have the following solutions.

$$
\begin{aligned}
& 27^{3}-28^{3}-\cdots-80^{3}+81^{3}=6^{7}, \\
& (-2)^{3}-(-1)^{3}+\cdots-5^{3}+6^{3}=2^{7}, \\
& (-12)^{3}-(-11)^{3}+\cdots-17^{3}+18^{3}=3^{7}, \\
& (-14)^{3}-(-13)^{3}+\cdots-25^{3}+26^{3}=6^{5} .
\end{aligned}
$$

This concludes the proof of Theorem 1.1 for $p \geq 5$.

\section{Proof of Theorem 1.1 For $p=2$}

Putting $x+d+1=u$ and $p=2$ in the equation (1.11), we have

$$
z^{2}=u^{3}+3 d(d+1) u .
$$

This represents a family of elliptic curves. For $1 \leq d \leq 50$, we obtain the integral solutions of the equation (4.1) by Magma ([4]). These solutions give rise to all the integral solutions of (1.11) and those are given explicitly in Table 1 .

\section{Proof of Theorem 1.1 for $p=3$}

In this case the required equation is

$$
z^{3}=u^{3}+3 d(d+1) u .
$$

Let $\alpha=\operatorname{gcd}(u, 3 d(d+1))$, then

$$
u=\alpha u_{1} \text { and } u^{2}+3 d(d+1)=\alpha u_{2},
$$

where $\operatorname{gcd}\left(u_{1}, u_{2}\right)=1$. Let $\operatorname{Ord}_{2}\left(u_{1} u_{2}\right)=l$ and $\operatorname{Ord}_{3}\left(u_{1} u_{2}\right)=m$, for some non-negative integers $l, m$. Then we can write

$$
\begin{aligned}
u_{1} & =2^{l} \cdot u_{3} \text { and } u_{2}=3^{m} \cdot u_{4}, \\
\text { or } u_{1} & =3^{l} \cdot u_{3} \text { and } u_{2}=2^{m} \cdot u_{4}, \\
\text { or } u_{1} & =2^{l} \cdot 3^{m} \cdot u_{3} \text { and } u_{2}=u_{4}, \\
\text { or } u_{1} & =u_{3} \text { and } u_{2}=2^{l} \cdot 3^{m} \cdot u_{4},
\end{aligned}
$$

where $u_{3}$ and $u_{4}$ are integers with $\operatorname{gcd}\left(u_{3}, u_{4}\right)=1$.

Also write $\alpha=2^{\delta_{2}} \cdot 3^{\delta_{3}} \cdot \alpha_{1}$ for some integer $\alpha_{1}$ with $\delta_{2}:=\operatorname{ord}_{2}(\alpha)$ and $\delta_{3}:=$ $\operatorname{ord}_{3}(\alpha)$. As $\alpha u_{1} \cdot \alpha u_{2}=z^{3}$, we have $\alpha_{1}^{2} u_{3} u_{4}=z_{1}^{3}$ for some integer $z_{1}$. Since $1 \leq d \leq 50$, for any prime $q \mid \alpha_{1}$, ord ${ }_{q}\left(\alpha_{1}\right) \leq 2$. Therefore, we can conclude 
that, if $\alpha_{1}^{2} \mid z_{1}^{3}$ then $\alpha_{1} \mid z_{1}$. Write $z_{1}=\alpha_{1} \cdot z_{2}$ for some integer $z_{2}$, hence we have $u_{3} \cdot u_{4}=\alpha_{1} z_{2}^{3}$. Since $\operatorname{gcd}\left(u_{3}, u_{4}\right)=1$, we can write

$$
u_{3}=\alpha_{2} \cdot z_{3}^{3} \text { and } u_{4}=\alpha_{3} \cdot z_{4}^{3}
$$

for some integers $\alpha_{2}, \alpha_{3}, z_{3}, z_{4}$ with $\alpha_{2} \alpha_{3}=\alpha_{1}$ and $z_{3} z_{4}=z_{2}$. Rewriting the equation (5.2), we have

$$
\alpha \cdot u_{2}-\alpha^{2} \cdot u_{1}^{2}=3 d(d+1) .
$$

Now from equations (5.3),(5.4) and (5.5), we will have a set of Thue equations as follows:

$$
\begin{gathered}
\alpha \cdot \alpha_{3} \cdot 3^{m} \cdot z_{4}^{3}-\alpha^{2} \cdot \alpha_{2}^{2} \cdot 2^{2 l} \cdot\left(z_{3}^{2}\right)^{3}=3 d(d+1), \\
\text { or } \alpha \cdot \alpha_{3} \cdot 2^{m} \cdot z_{4}^{3}-\alpha^{2} \cdot \alpha_{2}^{2} \cdot 3^{2 l} \cdot\left(z_{3}^{2}\right)^{3}=3 d(d+1), \\
\text { or } \alpha \cdot \alpha_{3} \cdot z_{4}^{3}-\alpha^{2} \cdot \alpha_{2}^{2} \cdot 2^{2 l} \cdot 3^{2 m} \cdot\left(z_{3}^{2}\right)^{3}=3 d(d+1), \\
\text { or } \alpha \cdot \alpha_{3} \cdot 2^{2 l} \cdot 3^{2 m} \cdot z_{4}^{3}-\alpha^{2} \cdot \alpha_{2}^{2} \cdot\left(z_{3}^{2}\right)^{3}=3 d(d+1) .
\end{gathered}
$$

Now, for $1 \leq d \leq 50$ we have written a Magma script ([4]) to solve these four Thue equations. The theory about solving these Thue equations is discussed in [19]. Using backward calculations from these solutions we find all solutions for the equation (5.1) and these are given explicitly in Table 1.

\section{CONCLUDING REMARK}

For $m=2 d$ the equation (1.9) becomes

$$
d\left[3 x^{2}+3(2 d+1) x+d(4 d+3)\right]=(-z)^{p} .
$$

Since the polynomial $3 d x^{2}+3 d(2 d+1) x+d^{2}(4 d+3)$ is an irreducible polynomial over $\mathbb{Q}$ for $1 \leq d \leq 50$, by [6, Theorem 12.11 .2 , p. 437], we conclude that the equation (1.9) has finitely many solutions for even $m$.

For $x>50$, we are not able to conclude anything about getting perfect powers in alternating sums of consecutive cubes of even length. Though for $x \leq 50$ we see that $(2,2,-2,7),(3,12,-3,7)$ and $(6,14,-6,5)$ are solutions for $(d, x, z, p)$ in the equation (1.9). In general, when $m$ is even in the equation (1.9), we conjecture the following.

Conjecture 6.1. Let $m=2 d$ with $1 \leq d \leq 50$ and $p \geq 5$ be a prime. Then the only integer solutions of the equation (1.9) are given by

$$
(d, x, z, p) \in\{(2,2,-2,7),(3,12,-3,7),(6,14,-6,5),(27,215,-9,7)\} .
$$

\section{ACKNOWLEDGEMENTS.}

We would like to thank Kalyan Chakraborty, Shanta Laishram and N. Saradha for their comments and suggestions on this problem. We also thank Samir Siksek and Manoj Kumar Yadav for their help related to this problem. 


\section{REFERENCES}

[1] M. Bennett, K. Győry and Á. Pintér, On the diophantine equation $1^{k}+2^{k}+\cdots+x^{k}=$ $y^{n}$, Compos. Math. 140 (2004), 1417-1431.

[2] M. Bennett, A superelliptic equation involving alternating sums of powers, Publ. Math. Debrecen 79 (2011), 317-324.

[3] M. Bennett, V. Patel and S. Siksek, Perfect powers that are sums of consecutive cubes, Mathematika 63 (2017), 230-249.

[4] W. Bosma, J. Cannon and C. Playoust, The Magma algebra system. I. The user language, J. Symboli Comput. 24 (1997), 235-265.

[5] J. W. S. Cassels, A Diophantine equation, Glasgow Math. J. 27 (1985), 11-88.

[6] H. Cohen, Number theory vol. II: analytic and modern tools, Springer, New York, 2007.

[7] K. Dilcher, On a Diophantine equation involving quadratic characters, Compos. Math. 57 (1986), 383-403.

[8] M. Jacobson, Á. Pintér, P. G. Walsh, A computational approach for solving $y^{2}=$ $1^{k}+2^{k}+\cdots+x^{k}$, Math Comp. 72 (2003), 2099-2110.

[9] A. Kraus, Majorations effectives pour l'équation de Fermat généralisée, Canad. J. Math. 49 (1997), 1139-1161.

[10] M. Laurent, Linear forms in two logarithms and interpolation determinants II, Acta. Arith. 133 (2008), 325-348.

[11] W. J. Leveque, On the equation $y^{m}=f(x)$, Acta. Arith. 9 (1964), 209-219.

[12] É. Lucas, Problem 1180, Nouvelle Ann. Math. 14 (1875), 336.

[13] B. Mazur, Rational isogenies of prime degree, Invent. Math. 44 (1978), 129-162.

[14] Á. Pintér, On the power values of power sums, J. Number Theory 125 (2007), 412-423.

$[15]$ K. Ribet, On modular representations of $\operatorname{Gal}(\overline{\mathbb{Q}} / \mathbb{Q})$ arising from modular forms, Invent. Math. 100 (1990), 431-476.

[16] J. J. Schäffer, The equation $1^{p}+2^{p}+\cdots+n^{p}=m^{q}$, Acta Math. 95 (1956), 155-189.

[17] A. Schinzel and R. Tijdeman, On the equation $y^{m}=f(x)$, Acta Arith. 31 (1976), 199-204.

[18] S. Siksek, Modular approach to Diophantine equations, in: Explicit methods in number theory, Panor. Synthèses 36, Soc. Math. France, Paris, 2012, 151-179.

[19] N. P. Smart, The algorithmic resolution of Diophantine equations, Cambridge University Press, 1997.

[20] G. Soydan, On the Diophantine equation $(x+1)^{k}+(x+2)^{k}+\ldots+(l x)^{k}=y^{n}$, Publ. Math. Debrecen 91 (2017), 369-382.

[21] R. J. Stroeker, On the sum of consecutive cubes being a square, Compos. Math. 97 (1995), 295-307.

[22] G. N. Watson, The problem of the square pyramid, Messenger of Math. 48 (1918), $1-22$.

[23] A. Wiles, Modular elliptic curves and Fermat's Last Theorem, Ann. Math. (2) 141 (1995), 443-551.

[24] Z. Zhang and M. Bai, On the diophantine equation $(x+1)^{2}+(x+2)^{2}+\cdots+(x+d)^{2}=y^{n}$, Funct. Approx. Comment. Math. 49 (2013), 73-77.

[25] Z. Zhang, On the Diophantine equation $(x-1)^{k}+x^{k}+(x+1)^{k}=y^{n}$, Publ. Math. Debrecen 85 (2014), 93-100. 
P. Das

Pure Mathematics, University of Waterloo

200 University Avenue West

Waterloo, Ontario

Canada

E-mail: pranabesh.math@gmail.com

P. K. Dey

Stat-Math Unit, Indian Statistical Institute

7, S. J. S. Sansanwal Marg, New Delhi, Delhi - 110016

India

E-mail: pallabkantidey@gmail.com

B. Maji

Department of Mathematics, Indian Institute of Technology Indore

Simrol, Indore, Madhya Pradesh - 453552

India

E-mail: bibekanandamaji@iiti.ac.in

S. S. Rout

Institute of Mathematics and Applications

Andharua, Bhubaneswar, Odisha - 751029

India

E-mail: lbs.sudhansu@gmail.com

Received: 9.7.2019. 